\title{
La didáctica no parametral: hacia un nuevo paradigma en la formación de docentes de inglés
}

\author{
Marcela del CAmpo M. \\ Departamento de Lenguas Extranjera, Universidad Nacional de Colombia
}

Recibido: 26 de junio de 2011 / Aceptado: 15 de septiembre de 2011

ISSN: $1697-7467$

\begin{abstract}
RESUMEN: Este artículo considera a la didáctica no parametral como un nuevo paradigma en la formación de docentes de inglés. Dicha didáctica construye o recrea formas de enseñar y aprender el inglés como lengua extranjera, al igual que desarrolla aspectos de importancia como la autonomía, la creatividad, y la construcción de conocimiento, entendido éste como una construcción de sentidos y significados, entre otros.

El artículo aborda los objetivos y los elementos que constituyen dicha didáctica, y luego se adentra en dos propuestas alternativas y/o complementarias al uso de los libros de texto los cuales han sido entendidos como el eje fundamental en la enseñanza del inglés desde la didáctica tradicional o parametral.
\end{abstract}

Palabras clave: Didáctica no parametral, autonomía, propuestas alternativas

\begin{abstract}
Non-parametric didactics as a new paradigm in the training of English teachers
\end{abstract}

\begin{abstract}
The aim of this article is to help understand non-parametric didactics as a new paradigm in the training of English teachers. Non-parametric didactics builds or recreates new ways of teaching and learning English as a foreign language, while developing other important aspects like autonomy, creativity and the construction of knowledge, involving the construction of sense and meaning, among other aspects. This article approaches the objectives and elements making up this type of didactics and then analyzes in depth two alternative and/or complementary proposals to the use of textbooks, which have been the fundamental axis of English teaching within the traditional or parametric didactics framework.
\end{abstract}

Keywords: Non-parametric didactics, autonomy, complementary proposals

\section{LA didÁCtica No PARAMETRAL: unA PEDAgogía de la POTENCIA}

Esta didáctica es una propuesta realizada por Quintar (2002), pensadora argentina para quien la base fundamental de la pedagogía es la de re-crear formas de enseñar, y formas de aprender. Para ella, el único punto común entre la didáctica parametral y no parametral es el horizonte de sentido de las dos, es decir, la enseñanza. La didáctica no parametral es un esfuerzo por generar una propuesta alternativa de ruptura con lo establecido por la lógica dominante que configura el sistema educativo. 
Esta autora la define en otras palabras así:

La didáctica no-parametral es una postura que comprende el proceso de enseñanza como un proceso intencional de permanente promoción de ruptura de sentidos y significados en el propio devenir existencial; lo que implica definir:

- Al conocimiento como construcción de sentidos y significados, es decir, de redes de representaciones simbólicas históricas e historizadas.

- Al sujeto concreto como sujeto atado a su territorialidad contextual y a su subjetividad, lo que hace de él un sujeto dialéctico en tanto que se articula en su sujeción simbólica, como sujeto de deseo y como sujeto del inconsciente, sujeciones que se actúan en el mundo de relaciones situadas, en este caso, en situaciones de enseñanza aprendizaje concretas (Quintar, 2002:12).

De acuerdo con esta misma autora, las estructuras no parametrales permiten vivenciar una realidad dinámica en la que los sujetos logran establecer vínculos y se recobra el sentido de una práctica docente que responda a la realidad de los alumnos.

Dado lo anterior, esta didáctica tendría como propósito desarrollar el pensamiento de los sujetos, enseñar a pensar y permitir que el aprendizaje sea entendido como una de las maneras de desarrollar el pensamiento; de esta forma se formarían sujetos con un pensamiento autónomo, libre de parámetros, que desarrollen en los estudiantes procesos de articulación para la construcción de nuevos conocimientos. Según Quintar (2002), se quiere formar personas que posibiliten procesos transformativos de aprendizaje a través de la promoción del deseo de saber y de expresar ese saber, problematizando su realidad.

En síntesis, la didáctica no parametral busca romper paradigmas establecidos que ponen límites a nuestras actuaciones y dependencias como docentes, y emprender la búsqueda de una creatividad intelectual para formar sujetos críticos, sin esquemas ya establecidos. En palabras de Quintar (2002) la didáctica no parametral «es una postura que comprende el proceso de enseñanza como un proceso intencional de permanente promoción de ruptura de sentidos y significados en el propio devenir existencial» (Quintar, 2002:16).

\subsection{Objetivos de la didáctica no parametral}

La didáctica no parametral tiene como propósito fundamental desarrollar en los estudiantes procesos de articulación para la construcción autónoma de nuevos conocimientos. Quintar (2002) lo expresa de la siguiente manera: «con la didáctica no parametral se pretende formar sujetos que posibiliten procesos transformativos de aprendizaje a través de la promoción del deseo de saber y de expresar ese saber, problematizando su realidad» (Quintar, 2002:18).

Para lograr lo anteriormente expuesto, la autora retoma conceptualizaciones dadas ensu propuesta, y que se plasman en el texto La enseñanza como puente a la vida; y son:

- La posibilidad de contextuar a los sujetos que aprenden.

- La construcción social del conocimiento.

- La participación real. 
- La formación de un pensamiento libre.

- La reflexión constante de lo que se enseña y se aprende.

- El planteamiento permanente de la duda.

- El reconocimiento de la educación como práctica social intencional.

- La creatividad como medio para resolver lo cotidiano.

- El mundo y lo que en él hay de enriquecedor.

Lo expresado implica una propuesta de didáctica dinámica en consonancia con la realidad dinámica del mundo, es decir, se trata de recuperar la exigencia de que las cosas están en constante movimiento, que la realidad nunca es estática ni definida.

De acuerdo a lo anterior, el papel del profesor vendría dibujado por una curva ascendente entre dos ejes (cuya ordenada fuera la sucesión temporal y la abscisa el grado de intervención), que iría desde un rol de mero gestor de prescripciones externas a «agente de desarrollo particular», pasando por un papel mediador en el proceso de innovación.

Lo anterior puede interpretarse como que existe una lógica de mayor desarrollo autonómico en la medida en la que crece mental y físicamente el alumnado, o en otras palabras, se produce una relación inversamente proporcional entre la formación académica y en el caso particular de la didáctica tradicional en lo relacionado con la dependencia de los libros de texto.

\subsection{La autonomía como elemento esencial de la didáctica no parametral}

Este apartado plantea el desarrollo de la autonomía en el aprendizaje del inglés como factor fundamental de la didáctica no parametral. Este elemento entreteje el desarrollo de otros aspectos, como el aprendizaje colaborativo, la motivación, la confianza, y la creatividad entre otros, que contribuyen a hacer de este tipo de didáctica una pedagogía de la potencia.

En primer lugar el concepto de autonomía ha sido desarrollado por diversos autores como Holec (1981:3) para quien ésta es «the ability to take charge of one's learning». Scharle y Szabó (2000), y Boud (1995), por su parte, desarrollan un poco más esta idea y dicen que la autonomía no sólo es la habilidad para asumir la responsabilidad de nuestro propio aprendizaje, sino que acarrea la responsabilidad de tomar las decisiones apropiadas para tal fin de manera que los individuos se perciban a sí mismos como los artífices de su propio aprendizaje (Fazey y Fazey, 2001).

Camilleri (2006) y Holliday (2003) involucran de manera acertada el aprendizaje social como parte del desarrollo de la autonomía; el primero argumenta que «learner autonomy is a social process in the sense that interaction with colleagues and others is essential», y el segundo complementa esta idea al plantear que la autonomía reside en los mundos sociales de los estudiantes los cuales son inherentes a sus vidas fuera del salón de clases; este aprendizaje social es fundamental en la medida en que es a través del otro que se reflexiona e interactúa para lograr una sinergia en la que se asumen los propios ritmos y se identifican las potencialidades que luego llevan a un aprendizaje autónomo.

Todos coinciden en que la autonomía es una habilidad con la que los individuos no nacen, sino que es una habilidad que debe ser desarrollada natural o formalmente. En este 
trabajo se hará referencia a la segunda posibilidad, es decir, al desarrollo de la autonomía de manera formal. Al hacer referencia al término formal, se hace referencia al desarrollo de la autonomía dentro del aula como tarea asignada al profesor. Sin embargo, la autonomía del aprendizaje, quiere plantearse en este escrito como un proceso que tiene lugar tanto dentro como fuera del aula, y que desde la didáctica no parametral se nos invita a los docentes a replantearnos formas de recrear la enseñanza y el aprendizaje necesarios para su desarrollo.

En este sentido, es necesario entender lo que el desarrollo de la autonomía supone. Wenden (1991) plantea que "'Successful' or 'expert' or intelligent learners have learnt how to learn. They have acquired the learning strategies, the knowledge about learning, and attitudes that enable them to use these skills and knowledge confidently, flexibly, appropriately and independently of a teacher. Therefore they are autonomous» (Wenden, 1992:15). Otros, como Martín Peris (2010), plantean que hay aspectos necesarios que deben tenerse en cuenta para desarrollar la autonomía como son la negociación, la autoevaluación y el desarrollo de técnicas y estrategias de aprendizaje y comunicación.

En este trabajo se propone el desarrollo de los siguientes elementos para el desarrollo de la autonomía:

Motivación y confianza en sí mismo: la motivación es un elemento esencial para que los estudiantes tomen responsabilidad de su propio aprendizaje, en especial es importante el desarrollo de la motivación intrínseca la cual se abordó ampliamente en este capítulo. Igualmente, la confianza en sí mismo contribuye al desarrollo de la responsabilidad de cada individuo dado que ellos deben creer que son capaces de tomar control de su propio aprendizaje, y que pueden confiar en sí mismos y no solamente en el profesor.

Seguimiento y evaluación: se hace necesario brindarle a los estudiantes las herramientas necesarias para que ellos sean capaces de evaluar su contribución en el proceso de aprendizaje. Para lograrlo, es importante involucrar procesos de autoevaluación de manera que ellos aprendan tanto a reconocer sus fortalezas y debilidades y planeen estrategias para redireccionar su progreso como a volverse más independientes e incrementar su motivación.

Estrategias de aprendizaje: Mora (2008) plantea que la autonomía es la creación o potenciación de estrategias de aprendizaje individuales en el alumno; éstas hacen referencia a las maneras o métodos que los estudiantes utilizan para aprender y pueden variar desde técnicas para mejorar la memoria hasta estrategias para evaluar sus progresos. Entre las estrategias que algunos autores mencionan están, entre otras, aquellas que se relacionan con la resolución de problemas como el entrenamiento de ciclo doble o aprendizaje bidireccional (double loop learning) propuesto por Argyris (1993), o el pensamiento lateral propuesto por DeBono (1970).

Lo más relevante es la labor realizada con los estudiantes al mostrarles la variedad de estrategias que existen y cuáles podrían ayudarles a mejorar su proceso de aprendizaje. De la misma manera es importante ayudarles a descubrir cómo y cuándo utilizarlas.

Trabajo colaborativo: el trabajo en pequeños grupos es una forma de lograr que estudiantes con distintos niveles de conocimientos y habilidades usen una variedad de actividades de aprendizaje para mejorar su comprensión respecto a algún tema específico. Cada individuo debe ser responsable no sólo de su propio aprendizaje sino de ayudarle 
a sus compañeros a aprender resultando en un beneficio mutuo. Boud (1995) agrega que este tipo de trabajo es importante para desarrollar la interdependencia la cual es una característica fundamental de los procesos de autonomía.

Delegación de tareas y decisiones: este aspecto es fundamental para que los estudiantes ejerzan gran influencia en su aprendizaje; por lo tanto es conveniente que ellos no sientan miedo de cometer errores y por el contrario, es primordial que sientan que son parte esencial de su aprendizaje.

Finalmente, es importante tener en cuenta que la autonomía no es una fase mágica, por el contrario puede considerarse un proceso gradual de formación en el que el aprendiz adquiere independencia en su proceso de aprendizaje. De manera similar, es relevante anotar que la autonomía es un elemento indispensable en el desarrollo e implementación de las propuestas de la didáctica no parametral.

\section{Propuestas alternativas y/o complementarias}

Este apartado presenta dos propuestas innovadoras desde la didáctica no parametral que buscan complementar el trabajo que se lleva a cabo con el libro de texto en el aula, entendiendo este último como una herramienta importante para el aprendizaje del inglés.

Dichas propuestas consideran que la lengua es una construcción social que se manifiesta individualmente y que sirve para crear emociones y compartirlas con los demás, y por lo tanto ha de enseñarse aprovechando al máximo su poder creador.

De igual manera se enmarcan dentro de esta didáctica porque, entre otras, son propuestas dinámicas que pretenden:

- Recrear formas de enseñar y aprender: este elemento es fundamental para la didáctica no parametral porque lo que pretende es buscar nuevas maneras de entender y propiciar el aprendizaje y la enseñanza.

- Vivir el conocimiento como una construcción de sentidos y significados: al brindarle al estudiante posibilidades de aprender de una manera significativa, con elementos de su entorno, y con sus compañeros; todo aquello que el estudiante construya adquirirá un nuevo sentido y significado para él.

- Romper con la concepción de límites, prejuicios y estereotipos: al ser propuestas didácticas que se realizan no sólo dentro del aula, sino fuera de ella, se rompe con los esquemas establecidos por la didáctica tradicional donde la enseñanza y el aprendizaje deben llevarse a cabo en un salón de clases.

- Formar futuros docentes de lengua extranjera que posibiliten procesos transformativos de aprendizaje a través de la promoción del deseo de saber, y de expresar ese saber problematizando su realidad.

- Fomentar el trabajo colaborativo: caracterizado este último por la igualdad que debe tener cada estudiante en el proceso de aprendizaje, y la mutualidad, entendida como la conexión, profundidad y bidireccionalidad que alcance la experiencia, siendo ésta una variable en función del nivel de competitividad existente, la distribución de responsabilidades, la planificación conjunta y el intercambio de roles (Díaz Barriga, 2003). 
- Desarrollar en los estudiantes procesos de articulación para la construcción autónoma de nuevos conocimientos: entendiéndose la construcción autónoma de nuevos conocimientos como un proceso continuado de inclusión que permite el crecimiento, elaboración y modificación de los conceptos debido a la adición de nuevos conceptos.

- Vivir la creatividad como medio para resolver lo cotidiano: la creatividad entendida como la posibilidad de tener en los estudiantes, agentes de cambio, capaces de enfrentar los retos de una manera diferente y audaz.

- Fomentar la unión de lo teórico y lo práctico: porque en el momento de llevar a cabo las propuestas, los estudiantes deben poner en práctica todo lo aprendido: gramática, pronunciación, entonación, etc.

- Desarrollar competencias de aprendizaje y otras propuestas por el MCER como la competencia lingüística, la sociolingüística y la pragmática.

- Propiciar el pensamiento autónomo: a través del fomento de la motivación y confianza en sí mismos, monitoreo y evaluación constante y el aprendizaje de estrategias como resolución de problemas.

Luego de haber resumido los aspectos que hacen de estas propuestas didácticas, propuestas desde la didáctica no parametral, se describirán cada una de ellas, resaltando los elementos más importantes que las constituyen.

Se presentarán las propuestas denominadas: desarrollo de la competencia comunicativa a través de la elaboración de videos, y creación de canciones: de la experiencia al aprendizaje a través de la literatura, las cuales tienen como base la literatura.

\subsection{Desarrollo de la competencia comunicativa a través de la elaboración de videos}

Dado que en las clases de lengua extranjera se experimenta la carencia de propuestas desde la didáctica no parametral que promuevan a partir de la literatura, la competencia comunicativa, desarrollen la autonomía, y que al mismo tiempo creen una actitud positiva por parte de los alumnos hacia el aprendizaje, se presenta en este aparte una propuesta alternativa que permita al estudiante involucrarse en la literatura como un ser activo haciendo uso real del idioma, y una propuesta en donde se desarrollen como ejes vitales la motivación, la participación, el trabajo en grupo, el desarrollo de la imaginación, el estímulo por la lectura y la escritura como claves fundamentales en el aprendizaje creando verdaderas comunidades de aprendizaje que son entendidas como «contextos en que los que los alumnos aprenden gracias a su participación e implicación en colaboración con otros alumnos, con el profesor y otros adultos en procesos genuinos de investigación y construcción colectiva de conocimiento sobre cuestiones personales y socialmente relevantes» (Pulido y Pérez, 2004:20).

Se quiere que los estudiantes piensen, diseñen, creen y elaboren un video con el ánimo de comunicarse; así, se les proveerían las facilidades para conducirlos a un medio en donde ellos puedan expresarse de acuerdo con sus necesidades individuales y de grupo, de manera que se involucren en la competencia lingüística. 
Lo más importante es poner la producción del video en sus manos y dejar que ellos actúen por completo desde que comienzan con la lectura de la historia hasta que se presenta el producto final. Esta actuación permite que los estudiantes muestren su capacidad de expresión y comunicación bajo condiciones que están lo más cerca posible de aquellas que puedan darse en una comunicación normal.

En conclusión puede afirmarse que las siguientes son las razones que hacen pensar en el uso de esta estrategia metodológica:

1. Porque es una forma de recrear formas de enseñar y aprender.

2. Porque es una posibilidad de acercar a los estudiantes para que no sólo conozcan la literatura y sus autores, desarrollen la competencia lectora, y las capacidades de análisis de textos, sino que la vivan, la recreen, la sientan y la disfruten.

3. Es la oportunidad que tienen los estudiantes de escuchar, hablar, escribir y leer usando la lengua dentro de un contexto real.

4. Porque los estudiantes desarrollan la competencia comunicativa en un espacio diferente a los comúnmente utilizados.

5. Porque desarrollan otras competencias como la de aprendizaje.

6. Porque desarrollan procesos de autonomía en el aprendizaje.

Puede plantearse entonces que esta propuesta no sólo quiere incrementar la competencia comunicativa de los estudiantes, sino que también pretende que los alumnos logren conocerse en actividades diferentes que los integren fuera del salón de clase, y que por supuesto los motive a utilizar la lengua que están aprendiendo fuera del espacio normal de ella. Esto fortalece sus vínculos sociales, los hace más responsables y logran superar muchas de las dificultades o resistencias hacia el aprendizaje de una lengua.

El proceso anterior exige de los estudiantes el diálogo, la interacción y la comunicación lo que los lleva a resignificar su concepción sobre el aprendizaje de la lengua y a construirse como sujetos sociales resaltando los principios de una verdadera comunidad de aprendizaje en donde se quiere «realzar la importancia de sus miembros, para que cada uno se sienta valorado, parte de una comunidad, reconocido como un miembro competente, donde sus necesidades son asumidas y donde puede experimentar placenteramente el proceso de aprendizaje» (Pulido y Pérez, 2004:3).

Es así como la elaboración de videos tiene como base el trabajo colaborativo en el cual según Calzadilla (2004:4) «cada participante asume su propio ritmo y potencialidades, impregnando la actividad de autonomía, pero cada uno comprende la necesidad de aportar lo mejor de sí al grupo para lograr un resultado sinérgico, al que ninguno accedería por sus propios medios». El desarrollo de este proyecto permite a los estudiantes interactuar como parte fundamental del aprendizaje fortaleciendo las relaciones interpersonales para lo cual es necesario que ellos se involucren en esta experiencia dentro y fuera del aula de manera que el aprendizaje de la lengua la trascienda.

\subsubsection{Propuesta del proyecto didáctico}

Este proyecto pretende mostrar cómo pueden ser desarrolladas propuestas desde la didáctica no parametral para el fomento de la creatividad, el desarrollo de las competen- 
cias comunicativas, el incremento de la autonomía, la promoción del trabajo colaborativo en la enseñanza de la lengua a través de la producción de un video, el cual se fundamenta en la lectura de una novela.

Igualmente, esta propuesta busca desarrollar el ejercicio de las cuatro habilidades porque brinda a los estudiantes la oportunidad de practicar la lengua oral, la lectura, la escritura, y la escucha, convirtiéndose en una experiencia real de comunicación, e interactuando genuinamente en un trabajo que los hace sujetos activos en la construcción y la producción del video. Adicionalmente, la elaboración de este proyecto desarrolla la autonomía como eje central de la didáctica no parametral.

\subsubsection{Pasos del proyecto didáctico: la elaboración del video}

A continuación se describirán algunos aspectos relevantes que deben seguirse en el proceso mencionado. Igualmente se describirá en qué consiste este proyecto grupal.

\subsection{Selección y lectura del libro}

Se procederá entonces a hacer una explicación del primer paso que es la lectura del libro. Este paso es muy importante ya que el desarrollo de las habilidades comunicativas comienza con la Interpretación de los textos (Cf. Savignon, 1983).

Como se mencionó anteriormente, lo primero es la organización de los grupos, aproximadamente de cuatro o cinco personas, y luego se lleva a cabo un consenso con los estudiantes respecto al libro que se leerá teniendo en cuenta el prestigio cultural del texto, y las expectativas de los estudiantes, entre otros. Es importante que ellos sepan que la lectura se hará de forma individual dentro y fuera del salón de clase. Una vez iniciado el proceso de lectura, los estudiantes se enfrentan a un trabajo de entendimiento del texto el cual tiene que ver con la competencia discursiva, la cual según Sandra Savignon se refiere a la habilidad de interpretar una serie de oraciones para formar un todo significativo y lograr textos coherentes que son relevantes en un contexto dado (Savignon, 1983:40).

Dado que la conexión entre las oraciones del texto en general, y del texto en particular, no es siempre explícita, el estudiante debe inferir el significado de los párrafos, basándose en la construcción de un nuevo conocimiento general del mundo que le es descrito en la lengua, a través del libro. Igualmente debe basar la construcción del significado a partir de la familiarización con los contextos que propone la lectura. De la misma manera, la interpretación del texto requiere una habilidad que le permita hacer una inferencia de sentido común de la situación mostrada por el autor. Este proceso estimula la imaginación y el intelecto de los alumnos.

Otro aspecto de relevancia para desarrollar en este primer paso es el enriquecimiento del vocabulario ya que los estudiantes de distintas maneras abordan palabras completamente nuevas para ellos. Por ejemplo, buscar palabras en el diccionario, interpretarlas a través del contexto o por conocimiento previo de la palabra, son algunas de las formas que el alumno tiene para acercarse a la inclusión de ésta a su léxico. El proceso va más allá de conocer el significado aislado de la palabra, va hacia la aplicación de ella en el momento de utilizarla en el diseño del libreto. 
El objetivo de este primer paso es proveer a los estudiantes de oportunidades de motivarse a sí mismos hacia el aprendizaje de una manera que podría llamarse significativa, ya que logra interesarlos y moverlos hacia la posibilidad de mejorar su competencia comunicativa.

\subsection{Discusión}

En este segundo paso los estudiantes dejan de trabajar individualmente e inician la realización de un trabajo grupal. Una vez establecidas las primeras pautas del trabajo, se espera favorecer la expresión oral en el sentido de desarrollar la fluidez, la elaboración, y la implicación personal.

En esta etapa del proceso, los alumnos deben discutir las generalidades de la construcción del video; es decir, hacer una interpretación general de las ideas principales que el texto les da, analizar y caracterizar los personajes que ellos consideran relevantes para el desarrollo de la historia y así escoger cuáles de ellos serán representados, cuáles omitidos y cuáles modificados o inventados.

De esta manera los estudiantes decidirán acerca de los ambientes adecuados para desarrollar el video y de la misma forma como hicieron con los personajes pensarán cuáles escenarios son los más acertados para la realización de la dramatización.

Puede decirse entonces que ésta es la fase de preparación para la representación en donde se deciden las actividades para realizar, se concretan los objetivos, se describe el escenario y los personajes, en donde este último es fundamental porque «la representación de los papeles es un tipo concreto de simulación que exige de los participantes una actuación o una representación dramática y que se centra en situaciones definidas por la interacción de unos individuos con otros» (Motos, 1999:93). Es importante decir que cuando un estudiante adopta un papel está asumiendo unos comportamientos apropiados para su rol específico y esto significa que él puede manifestarse de forma creativa y lúdica.

\subsection{Escribir el libreto}

La elaboración del video favorece la expresión escrita y por eso la relevancia de este paso. Fundamentalmente lo que se les pide a los estudiantes es que pongan en funcionamiento habilidades de pensamiento divergente que les permitan engrandecer el relato que hacen de la historia en el momento de re-escribirla. Es decir los alumnos deben convertir la idea que tienen de la historia en un guión que la narre creativamente. Ellos deben tener en cuenta que éste debe poseer cualidades como energía, proximidad y encarnación de los personajes debido a que éstos son claves en la creación y organización del libreto. En otras palabras, es necesario involucrarse e identificarse con el personaje escogido y esto depende de la cantidad de energía contenida en el relato de la historia.

La cristalización de este proceso evolutivo se ve reflejada en la escritura del libreto la cual está dada por algunas etapas. La primera es realizada en el salón de clase y consiste en escribir un primer bosquejo cuyo objetivo es la presentación de la historia en unos cuantos párrafos mostrando el desarrollo de ésta y la ubicación de la acción en el tiempo. En general mostrará la línea conductora del relato que será revisada por el profesor. 
La segunda etapa consiste en el tratamiento o partición secuencial de la historia, escena por escena que será perfeccionada en la elaboración del libreto final al cual serán agregados los diálogos, las descripciones y las narraciones. Esta tercera y última etapa es una serie de escenas que se entregaran al profesor para una última revisión.

Todo el proceso anterior busca que los estudiantes no sólo integren todo el conocimiento adquirido durante el desarrollo de la clase y que se ha denominado según Savignon (1983) como competencia gramatical o lingüística, sino que desarrollen su creatividad porque en la elaboración del video y específicamente en la escritura del libreto se reproducen en miniatura las fases del proceso creador las cuales se describirán brevemente a continuación:

a. La preparación que es donde el sujeto crea un clima favorable con los medios adecuados para juntar datos, liberar imágenes y visualizarlas lo cual se ve reflejado en la escritura del bosquejo.

b. La incubación que es la elaboración interna de la historia en donde se analizan las situaciones y se dan soluciones múltiples a los posibles problemas que surjan. Esta fase se refleja en el tratamiento de la obra.

c. La iluminación o plasmación de la idea total que se encontrará directamente encarnada en el libreto como tal.

d. La revisión que consiste en la evaluación de los resultados, la experimentación, la corrección y la puesta en práctica. Esta fase puede encontrarse en el proceso de retroalimentación entre profesor y estudiante el cual le permite a estos últimos pasar a la memorización del libreto y al inicio de la realización y puesta en escena de éste.

Se finaliza este paso resaltando la importancia de la competencia sociolingüística como campo interdisciplinario donde los estudiantes necesitan entender el contexto social en el que desenvuelve la historia y la forma en que ellos utilizan el lenguaje apropiado que corresponde a éste, asignándole a cada uno de los participantes sus roles, y recalcando la función de la interacción. Así, cada uno sabrá qué decir y cómo decirlo de acuerdo con las situaciones y contextos establecidos para la historia.

\subsection{Acción representada}

Este paso desarrollado en el salón de clase, busca que los estudiantes practiquen las líneas escritas por ellos e igualmente pretende que los mismos, a través de la práctica, logren mejorar su fluidez, pronunciación y entonación. En este momento juega un papel primordial el rol de los estudiantes más aventajados porque ellos promueven la monitorización, y la autocorrección de sus compañeros y de sí mismos generando el autocontrol de cada una de las personas involucradas en el grupo.

El estudiante comienza a ejercer un papel más activo debido a que en este momento se vuelve un actor cuyo principal objetivo es la comunicación en donde da forma a los mensajes que quiere hacer llegar al espectador. De esta manera la competencia estratégica se activa ya que es aquí cuando el estudiante debe utilizarla para compensar los límites que posea al momento de establecer la comunicación, por ejemplo, respecto al 
vocabulario, o a las mismas estructuras gramaticales. Así se aplicaría que una persona sólo aprende a comunicarse comunicándose.

Otro aspecto para resaltar de este paso es la recuperación de la palabra que ha sido desplazada por la cultura de la imagen. Los estudiantes a través de la dramatización y de la expresión oral llegarán a la expresión corporal la cual es fundamental como procedimiento didáctico para el aprendizaje de una lengua.

Es importante destacar la relevancia de darle vida a las líneas aprendidas como la posibilidad de permitir a los estudiantes proyectarse hacia situaciones o papeles imaginados. Por ejemplo, esta actuación exige de los participantes que acepten una serie de convenciones relativas a las conductas que se mantienen normalmente en las relaciones de unos con otros, a saber:

- Dejar en suspenso los roles sociales normales que se adoptan en las relaciones cotidianas con los otros, e identificarse con los nuevos roles imaginarios propuestos.

- Hacer un uso diferente del espacio y del tiempo.

El significado y la simbolización son también importantes en este paso ya que la dramatización «no es un mero hacer sino que este hacer tiene un significado simbólico» (Motos, 1999:148). Los estudiantes después de haber leído, escrito, diseñado y planificado la situación la representarán ante los demás, en este caso se realizará la filmación. Cada una de las situaciones seleccionadas funciona como un símbolo del tema, es decir, que al representar una idea abstracta de forma simbólica, el grupo la hace más concreta $\mathrm{y}$ de esa forma se clarifica su significado.

\subsection{Filmación}

Este último paso permite a los estudiantes la repetición de las escenas hasta lograr la mejor realización posible del video, donde rectifican errores gramaticales, mejoran la pronunciación y la entonación. Esta fase final del proyecto se hace de manera real, porque los alumnos se han apropiado tanto del tiempo como del espacio, es decir, su vestuario es el adecuado para la situación representada, el tiempo es el marco dentro del cual desenvolverse y el espacio son los escenarios escogidos por ellos con anterioridad. Los alumnos igualmente han internalizado sus líneas y se sienten plenamente identificados con sus roles, lo que les permite improvisar, como elemento necesario del lenguaje real.

Más aún, «la improvisación activa el conocimiento ya existente de la lengua y prueba las estrategias comunicativas de los estudiantes» (Wessels, 1987: 85). Mientras se filma la dramatización todos los imperfectos pueden ser mejorados, rectificados y corregidos para hacer del producto final un gran proyecto y lo más importante es que al final de este proceso los estudiantes deben haber mejorado sustancialmente su competencia comunicativa.

\subsection{Creación de canciones: de la experiencia al aprendizaje a través de la literatura}

Esta propuesta al igual que la anterior busca no sólo incrementar las habilidades comunicativas, sino propiciar el trabajo autónomo y colaborativo fuera del aula de manera que los estudiantes encuentren ambientes donde se sientan cómodos y libres para utilizar 
la lengua que están aprendiendo. Este trabajo requiere diálogo, interacción, y comunicación entre los estudiantes para que ellos le encuentren un nuevo sentido al aprendizaje de la lengua.

Así, esta propuesta asume los principios más importantes de las comunidades de aprendizaje donde se aprende del conocimiento del otro, se monitorean los objetivos de lo que se realiza para lograr las metas propuestas, se respetan la contribuciones de los demás, se aprende de los errores y se negocian las actividades por realizar dentro del grupo.

Como resultado, en palabras de Pulido y Pérez (2004) cada miembro del grupo es importante, valorado y reconocido como un igual, lo que permite que se disfrute el proceso de aprendizaje.

\subsubsection{Pasos del proyecto didáctico: Creación de canciones}

Este proyecto tiene en cuenta la importancia de conectar la literatura con la música dado que existe un valor íntimo que relaciona la música con el lenguaje porque los sonidos son las raíces de ambos (Fonseca, 2000). Así, lo anterior permite reconsiderar y tomar ventaja de la interacción entre la música y la adquisición del lenguaje. Ambas disciplinas comparten algunas características comunes como el tono, el volumen, la entonación y el ritmo, las cuales son aprendidas por exposición; lo que significa que ninguna lengua puede ser aprendida sin «input» oral o escrito.

Por otra parte, de acuerdo con la didáctica las canciones son útiles para enseñar el ritmo de la lengua y para inculcar en los estudiantes la cultura de la misma. Otro aspecto relevante, es que la música crea ambientes de aprendizaje relajados que mejoran o brindan mejores condiciones al proceso de aprendizaje (Murphey, 1990).

Además, es necesario fomentar la inteligencia corporal entre los estudiantes dado que se hace necesario explorar otras formas de aprender ya que todos lo hacemos de una manera diferente (Gardner,1983); por lo tanto, los estudiantes que no utilizan las maneras habituales de repetición coral, o ejercicios de repetición individual, pueden encontrar en el cantar una canción una manera sencilla de entender y memorizar algo (entendida la memorización como una habilidad cognitiva necesaria para el aprendizaje); es importante explotar el hecho de que la música no sólo parece dejar una huella imborrable en nuestras memorias sino que ayuda a los estudiantes a mejorar su pronunciación y la adquisición del lenguaje.

Teniendo en cuenta las ideas expresadas con anterioridad, se enumerarán los pasos a seguir para escribir la canción; estos pasos son desarrollados en el aula y fuera de ella:

\subsubsection{Selección del libro y discusión}

Esta propuesta se lleva a cabo en grupos de tres estudiantes y la meta principal es crear una canción a partir de la novela que hayan leído; es decir, la historia de la canción debe corresponder a la historia contada en la novela. El objetivo de esta etapa es que los estudiantes escojan la novela que van a leer de acuerdo con los criterios establecidos de común acuerdo con el docente. Una vez se lleva a cabo el proceso de lectura, los estudiantes en grupo discuten sobre qué parte de la historia van a escribir la canción, 
o si van a resumir la historia en la canción. En este paso, el trabajo colaborativo es de gran relevancia porque las contribuciones de cada miembro del grupo son importantes para la toma de decisiones; igualmente el proceso de discusión les permite libremente expresar sus ideas en la lengua extranjera.

\subsubsection{Escritura de la canción}

Este paso consiste en que los estudiantes plasmen sus ideas en oraciones que a su vez deben corresponderse con la melodía. Es decir, la idea es que cuenten la historia que se desarrolla en la novela con música y que los eventos más importantes de dicha historia tengan ritmo. De esta manera, en lugar de escribir resúmenes de las novelas leídas (como se haría desde la didáctica tradicional), los estudiantes escriben canciones acerca de ellas.

Como resultado, se les pide a los estudiantes que escriban una canción con base en la historia leída, teniendo en cuenta que ellos son libres de comentar, reescribir la historia u ofrecer sugerencias acerca de la manera en que escribirán la canción. Durante este paso, se espera que el alumnado revise, aplique y refuerce todos los conocimientos gramaticales y fonéticos adquiridos como vocabulario, entonación, ritmo, etc.

En esta etapa, los estudiantes necesitan trabajar en equipo para inventar las líneas que conformarán la canción la cual en principio debe respetar los acentos normales de las palabras, y deben también coincidir con el acento musical que ellos hayan escogido para tal fin. Es importante aclarar que los estudiantes pueden escoger la melodía de cualquier canción que exista en el mercado para ponerle la letra, o también tienen la opción de componerla (para aquellos que tienen dotes musicales). Asimismo, el alumnado debe prestar atención al buen sentido del ritmo para que todo compagine y termine siendo una representación cantada de lo que normalmente dirían si estuvieran hablando.

De esta manera, el grupo de estudiantes debe coincidir en sus ideas respecto a la melodía y a las ideas que van a plasmar en el papel, lo que les exige entusiasmo, planeación y organización al igual que los motiva a ser miembros activos de su proceso de aprendizaje. Así, los estudiantes logran alcanzar un nivel de confianza en ellos mismos al sentirse iguales en un proceso donde todos deben contribuir con sus ideas. Esto les permite sentirse motivados con el aprendizaje de la lengua

\subsubsection{Grabación}

Los estudiantes graban la canción fuera del aula, y la escuchan hasta que quede lo más cerca posible a lo que imaginaron que podría hacer. En esta etapa tienen la oportunidad de repetir la grabación cuantas veces sea necesaria de manera que la canción sea agradable, melódica, rítmica y que por supuesto resuma la historia leída. Los estudiantes presentan la canción a sus compañeros para ser co-evaluada utilizando criterios establecidos con anterioridad.

Cabe recalcar que establecer una relación entre la música y el aprendizaje de una lengua crea una atmósfera reconfortante y cálida que es necesaria para desarrollar actividades de composición o escritura, y de pronunciación porque el hecho de que los estudiantes repitan, combinen y canten las palabras tiene un efecto positivo en el nivel de adquisición de la lengua. Lo anterior permite que el aprendizaje sea un proceso vivo 
que brinda a los estudiantes la oportunidad de inventar y reinventar el lenguaje para ellos mismos, un lenguaje que comunica y transmite sus ideas y sentimientos (Silva, 2000).

Finalmente autores como Lems (2001) y Little (1983) reivindican la importancia de los estudios que muestran que la música, particularmente las canciones, ayudan a los aprendices de lenguas a adquirir vocabulario, estructuras gramaticales, expresiones idiomáticas; igualmente los ayuda a mejorar la ortografía y la pronunciación, a desarrollar las habilidades de comprensión y las habilidades lingüísticas inherentes a la escritura, la lectura, la escucha y el habla y por supuesto, les ayuda a expandir su conocimiento cultural. De la misma manera, McParland (2003) amplía lo anterior planteando que la combinación entre música y lenguaje fomenta un procesamiento de la información más profundo, e incrementa la comprensión de lectura fortaleciendo el proceso de aprendizaje.

\section{Consideraciones finales}

A continuación se enuncian algunas consideraciones finales respecto a las propuestas alternativas planteadas desde la didáctica no parametral, y elaboradas para resignificar y flexibilizar el uso del libro de texto en la enseñanza del inglés como elemento fundamental de la didáctica tradicional en la formación de docentes de inglés.

- Es importante contar con propuestas desde la didáctica no parametral que dinamicen el uso del libro de texto, complementando la función que cumple en las aulas.

- Este tipo de propuestas permite recrear formas de aprender y enseñar.

- Estas propuestas permiten desarrollar la autonomia, el trabajo colaborativo, y el desarrollo de la creatividad como ejes fundamentales del aprendizaje.

- Es importante realizar experiencias que maximicen el trabajo colaborativo en el cual cada participante asume su propio ritmo y potencialidades, impregnando la actividad de autonomía, pero cada uno comprende la necesidad de aportar lo mejor de sí al grupo para lograr un resultado sinérgico, al que ninguno accedería por sus solos medios.

- La realización de propuestas como las que se mencionarán en el próximo capítulo permitirá la vivencia del aprendizaje del inglés por parte de los estudiantes de manera reflexiva e interactiva y como parte de un proyecto de vida, ya que el aprendizaje no puede ocurrir en el vacío social, sino en la relación que se establece entre las personas del grupo dejando de lado lo que Calzadilla llama «observadores pasivos y receptores repetitivos» porque los estudiantes crean sus propias formas de acercase a la lengua extranjera y de vivirla de acuerdo con sus posibilidades y potencialidades.

- Las Propuestas desde la didáctica no parametral permiten que los docentes se abran a nuevas experiencias que deben transformar la experiencia educativa en impacto trascendente para la efectiva inserción social del individuo, en términos de sus capacidades y actitudes para la convivencia y la autorrealización personal, profesional y laboral, ya que de esta manera el aprendizaje trasciende el aula y se hace extensivo a la vida. 


\section{REFERENCIAS BIBLIOGRÁFICAS Y FUENTES CITADAS}

Argyris, C. (1993). Knowledge for Action. A guide to overcoming barriers to organizational change. San Francisco: Jossey Bass.

Boud, D. (1995). «Moving towards autonomy». En Boud, D. (ed.). Developing student autonomy in learning. New York: Nichols Publishing, p. 17.

Camilleri, A. (2006). «Learner Autonomy». En Modern Language Learning. Consultado por última vez el 3 de octubre de 2009, en http://ajte.education.ecu.edu.au/issues/PDF/351/Bal.pdf

Calzadilla, M. E. (2004). «Aprendizaje Colaborativo y tecnologías de la información y la comunicación». Revista Iberoamericana de Educación OEI.

DeBono, E. (1970). El Pensamiento Lateral. Barcelona: Paidós.

Díaz Barriga, F. (2003). «Cognición situada y estrategias para el aprendizaje significativo». Revista Electrónica de Investigación Educativa, 5 (2). Consultado el día 4 de noviembre de 2009 en: http://redie.ens.uabc.mx/vol5no2/contenido-arceo.html.

Fazey, D. and Fazey, J. (2001). "The potential for autonomy in learning studies in Higher Education» vol. 26 no 3, 345. Find your bearing and pick your way. Consultado por última vez el 20 de agosto de 2010 en http://www.letmelearn.org/wp-content/uploads/camilleri.pdf.

Fonseca, C. (2000). «Foreign language acquisition and melody singing». ELT Journal April, 2000 Vol. 54, pp. 146-152.

Gardner, H. (1993). Finding cognition in the classroom: an expanded view of human intelligence. Cambridge: Cambridge University Press.

Holec, H. (1981). Autonomy and foreign language learning. Oxford: Pergamon press.

Holliday, A. (2003). Social autonomy: Addressing the dangers of culturalism in TESOL. In Palfreyman \& Smith (eds.).

Lems, K. (2001). Using music in the adult ESL classroom [Electronic version]. Consultado por última vez en agosto 2 de 2009, en ERIC/NCLE Digests Web site.

Little, J. (1983). Using music in the adult ESL classroom (Versión electrónica). TESL Talk, 14 (4), 40-44.

McParland, R. (2000). Music to their ears. Consultado por última vez el 20 de octubre de 2009 en Memorial University Newfoundland Queen Elizabeth II Library database.

Martín Peris, E. (2010). La educación para la autonomía: un nuevo paradigma docente. Consultado por última vez el 4 de septiembre de 2010 en http://www.upf.edu/pdi/dtf/ernesto.martin/ archivos/articulos/educacio autonomia. pdf.

Mora, M. (2008). El papel del profesor en la autonomía del aprendizaje del alumno de español como lengua extranjera. Consultado por última vez el 2 de septiembre de 2010 en http:/ /cvc.cervantes.es/ensenanza/biblioteca_ele/asele/pdf/05/05_0217.pdf.

Motos, T. (1999). Creatividad dramática. Universidad Santiago de Compostela: Servicio de publicación e intercambio científico Campus universitario sur.

Murphey, T. (1990). Using music and song in the foreign language classroom. Consultado por última vez en septiembre 2 de 2008, en http://www.laits.utexas.edu/hebrew/music/music.html.

Pulido, A. y Pérez, V. (2004). Hacia un concepto de competencia comunicativa integral: un novedoso acercamiento a sus dimensiones. Consultado por última vez el 15 de julio de 2009 en http://www.pr.rimed.cu/sitios/Revista\%20Mendive/Num7/pdf/2.pdf.

Quintar, E. (2002). Pedagogía de la potencia y didáctica no parametral: Entrevista con Estela Quintar. Consultado por última vez el 10 de marzo de 2010 en http://tariacuri.crefal.edu.mx/ rieda/ene jun_2009/aula magna/aula magna art1_p3.htm. 
Quintar, E. (2002b). La enseñanza como puente a la vida. México: Universidad Nacional del Comahue, UPN.

Savignon, S. (1983). Communicative competence: Theory and classroom practice. California: Addison-Wesley publishing company.

Scharle A. and Szabó, A. (2000). Learner Autonomy. Cambridge: Cambridge university Press.

Silva, C. (2000). Recent theories of language acquisition in relation to a semantic approach in foreign language teaching. Madrid: Hispania, 73, pp.163-176.

Wenden, A. (1991). Learner strategies for learner autonomy. NJ: Prentice-Hall International.

Wessels, Ch. (1987). «From improvisation to publication on an English through drama course». ELT Journal, 45, pp. 230-236. 